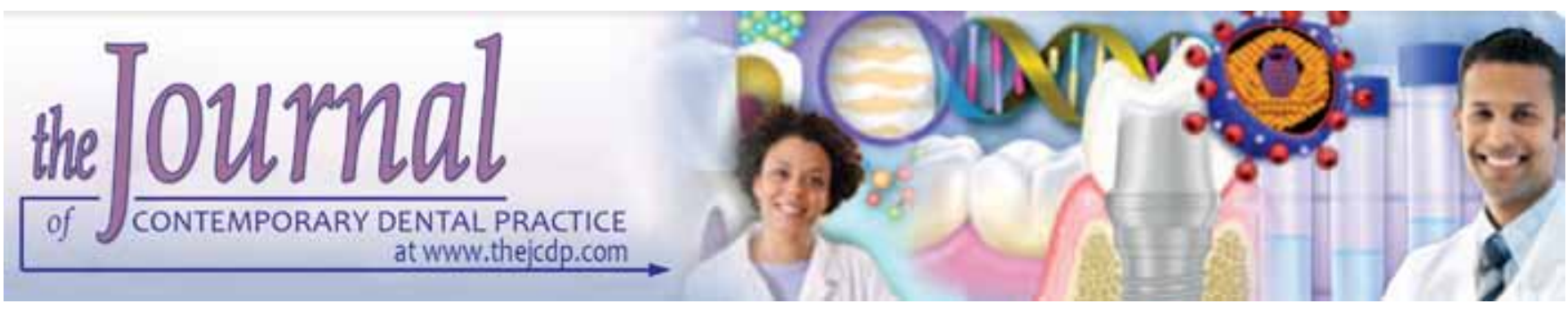

\title{
Evaluation of Gingival Inflammation in Patients using Ovulation Induction Drugs before and after Scaling
}

\author{
Sanjay Vasudevan, J Valli Renuka, D Sharon Sylvia, Radhika Challa, M Padmakanth, Ajay Reddy
}

\section{ABSTRACT}

Objectives: To determine and correlate the effect of clomiphene citrate, Letrozole in women undergoing infertility treatment on the gingival inflammatory status.

Materials and Methods: The present study is a randomized controlled clinical trial which consisted of 26 women using CC for three menstrual cycles, 26 women using $\mathrm{CC}$ for more than three cycles, 26 women using Letrozole. All subjects were clinically examined for plaque levels (Plaque Index), gingival inflammation, bleeding on probing (Gingival Index, Sulcus Bleeding Index). Scaling was done to all patients and all periodontal parameters were reassessed 1 month after scaling. The results were compared with a control group of 26 women matched for age, educational status and professional level, and oral habits and who had never used ovulation drugs.

Results: Baseline scores of all the test groups showed higher amount of plaque levels and inflammation compared to control. $(p<0.05)$. After scaling a significant reduction in inflammation was observed in all the test groups along with the control group ( $p<0.0001$ ), but women using the drugs showed persistence of inflammation compared to control ( $p<0.01)$.

Conclusion: It can be concluded from the present study that the presence of elevated levels of hormones due to the effect of ovulation induction drugs may be the reason for the gingival inflammation in test groups.

Keywords: Inflammation, Chlomiphene Citrate (CC), Letrozole, Scaling, Ovulation induction.

How to cite this article: Vasudevan S, Renuka JV, Sylvia DS, Challa R, Padmakanth M, Reddy A. Evaluation of Gingival Inflammation in Patients using Ovulation Induction Drugs before and after Scaling. J Contemp Dent Pract 2013;14(6):1165-1168.

\section{Source of support: Nil}

\section{Conflict of interest: None}

\section{INTRODUCTION}

Infertility is the inability of a couple to achieve conception after a year or more or regular, unprotected intercourse or incapacity to carry a pregnancy to live birth. ${ }^{1}$ Ovulation induction is a widely used method for infertility treatment not only for women with ovulatory disorders such as amenorrhea or anovulation but also for couples whose infertility is related count or defective sperm or unexplained factors. ${ }^{2}$ Various forms of ovulation induction agents used include Clomiphene citrate (CC), aromatase inhibitors, laparoscopic ovarian drilling, ovulation induction with Gonadotropins. ${ }^{3}$

The drugs used for ovulation induction result in depletion of serum estrogen and progesterone levels and sending signals to hypothalamus to release the gonadotropins that aid in development of follicles and aid in ovulation. ${ }^{4}$

Human gingiva have receptors for estrogen and progesterone and elevated levels of these hormones during puberty and pregnancy have all been associated with periodontal pathology. The mechanisms suggested to explain this phenomenon are diverse, and based on the observations that female sex hormones are capable of altering the gingival vascular system, depressing cell-mediated immune responses and modulating the subgingival flora. ${ }^{5-8}$

So, the present study was conducted to determine and correlate the effect of chlomiphene citrate, letrozole in women undergoing infertility treatment on the gingival inflammatory status.

\section{MATERIALS AND METHODS}

A group of hundred female subjects between the ages of 25 to 38 were included in this present study from Sri Balaji Nursing Home and also from Sahasra Multispeciality Dental Clinic, Hyderabad. All volunteers who participated in the study were provided written informed consent.

\section{INCLUSION CRITERIA}

Subjects who are infertile and undergoing treatment for infertility through ovulation induction, subjects with at least 25 teeth present in oral cavity, subjects with intermediate 
to graduate education, percapita income ranging between ₹ 10,000 to 30,000 .

\section{Exclusion Criteria}

Use of anti-inflammatory drugs in the past 3 months, pan and betel nut chewing, periodontal treatment within the previous 6 months, systemic conditions or diseases affecting the periodontium, patients with gingival over growth, gingival ulcerations or periodontal abscess.

\section{Study Population}

Subjects have been categorized into four (4) different groups according to the type of medication and duration of the subjects using medication as:

- Group A: Chlomiphene citrate $<3$ cycles

- Group B: Chlomiphene citrate $>3$ cycles

- Group C: Letrozole

- Group D: Control group (who did not use any of these medications).

\section{Study Design}

At the first visit the patients were interviewed about socioeconomic status, age, educational status and frequency of tooth brushing. For all the subjects the gingival index, plaque index and Sulcular bleeding index were recorded and a session of supragingival scaling was performed to all subjects irrespective of groups. The patients were taught oral hygiene measures and were advised to come after completion of 1 month for re-assessment of the indices noted previously.

The test and control groups were examined for plaque level, gingival inflammation and bleeding on probing. Measurements of all teeth except 3rd molars were recorded from four sites of each tooth that is mesio-buccal, midbuccal, disto-buccal and lingual/palatal. All measurements were performed with a manual periodontal probe (William's Periodontal Probe) by single examiner who was masked to control and study groups.

\section{RESULTS}

A total of hundred female patients undergoing infertility treatment participated in the study. All the subjects aged between 25 and 38 years mean age (27). Gingival index, plaque index, sulcus bleeding index scores were calculated on the day of examination as baseline and after 1 month after scaling.

Comparision of mean values obtained using paired t-test showed no significant differences among the test and control groups $(p>0.05)$ with higher index scores among test groups than control group.
Comparision of mean gingival index of various groups at baseline, I month postscaling were compared using Wilcoxon matched pairs test. The test showed significant decrease in the means of all groups between baseline, I month postscaling. All the values were statistically significant $(\mathrm{p}<0.05)$ (Table 1$)$.

Comparision of plaque index of various groups at baseline, I month postscaling were compared using Wilcoxon matched pairs test. The test showed significant decrease in the means of all groups between Baseline, I month postscaling. All the values were statistically significant $(\mathrm{p}<0.05)$ (Table 2$)$.

Comparison of sulcus bleeding index of various groups at baseline, I month postscaling were compared using Wilcoxon matched pairs test. The test showed significant decrease in the means of all groups between baseline, I month postscaling. All the values were statistically significant $(\mathrm{p}<0.05)$ (Table 3$)$.

\begin{tabular}{lllll}
\multicolumn{5}{c}{ Table 1: Comparison of gingival index before and after } \\
treatment
\end{tabular}

CC: Clomiphene citrate; ${ }^{* * *} \mathrm{p}$-value (statistical significance)

\begin{tabular}{lllll}
\multicolumn{4}{l}{ Table 2: Comparison of plaque index before and after treatment } \\
\hline Groups & Time & Mean & $S D$ & $p$-value \\
\hline CC $<3$ cycles & Day 0 & 1.3417 & 0.1558 & $0.00^{* * *}$ \\
& 1 month & 0.3542 & 0.1817 & \\
CC $<3$ cycles & Day 0 & 1.2792 & 0.2377 & $0.00^{* * *}$ \\
& 1 month & 0.3292 & 0.1899 & \\
Letrozole & Day 0 & 1.3417 & 0.1558 & $0.00^{* * *}$ \\
& 1 month & 0.3458 & 0.1978 & \\
Control & Day 0 & 1.0792 & 0.2859 & $0.00^{* * *}$ \\
& 1 month & 0.1875 & 0.1116 & \\
\hline
\end{tabular}

CC: Clomiphene citrate; ${ }^{* * *} \mathrm{p}$-value (statistical significance)

\begin{tabular}{lllll}
\multicolumn{5}{c}{ Table 3: Comparison of mean sulcus bleeding index } \\
before and after treatment \\
\hline Groups & Time & Mean & $S D$ & $p$-value \\
\hline CC $<3$ cycles & Day 0 & 52.1667 & 22.4745 & $0.00^{* * *}$ \\
& 1 month & 0.7500 & 2.1110 & \\
CC $>3$ cycles & Day 0 & 84.2083 & 8.7277 & $0.00^{* * *}$ \\
& 1 month & 1.8333 & 2.7767 & \\
Letrozole & Day 0 & 52.1667 & 22.4745 & $0.00^{* * *}$ \\
& 1 month & 1.0000 & 1.9337 & \\
Control & Day 0 & 26.8333 & 10.3867 & $0.00^{* * *}$ \\
& 1 month & 0.0000 & 0.0000 & \\
\hline
\end{tabular}

CC: Clomiphene citrate; ${ }^{* \star *} \mathrm{p}$-value (statistical significance) 


\section{DISCUSSION}

The role of bacteria on the initiation and progression of inflammatory periodontal diseases is well documented in the literature, so it was important for this study to eliminate its effects on the periodontium by exactly matching the test and control groups in terms of age, socioeconomic level, education level and oral hygiene habits. In this study, the gingival inflammation levels of women undergoing infertility treatment and subjected to ovulation induction drugs such as chlomiphene citrate (CC) and Letrozole which are the two major drugs used in ovulation induction, were investigated and compared with women who were not using these drugs. The effects of these drugs on gingiva before and after scaling were investigated.

Researchers have shown that changes in periodontal conditions might be associated with variations in sex hormone levels. This association is evident in the recent periodontal disease classification which includes the following hormone-related disease categories: pubertyassociated gingivitis, menstrual-cycle associated gingivitis and pregnancy associated gingivitis. The steroid sex hormones such as estrogen and estradiol have been known for their effect on bone mineral metabolism. ${ }^{9}$

Sex steroid hormones have been shown to directly and indirectly exert influence on cellular proliferation, differentiation and growth in target tissues, including keratinocytes and fibroblasts in the gingiva. Estrogen may be responsible for alterations of homeostasis of epithelium, connective tissue, vascularity, and production of keratin and collagen. Indeed, during pregnancy, the epithelial cell function is depressed and as a result the degree of keratinization of the gingiva is likely to be decreased. There is also an alteration in collagen metabolism with depressed rates of collagen synthesis. Estrogen and progesterone, act in the connective tissue inducing fibroblast proliferation and collagen maturation. Noncollagenous connective tissue proteins, such as glycosaminoglycan's are more rapidly degraded in tissues with elevated levels of both estrogen and progesterone. Progesterone alters the rate and pattern of collagen production in the gingiva, resulting in reduced repair and maintenance potential, resulting in an altered collagen turnover. ${ }^{10}$ Progesterone increases the permeability of blood vessels in target tissues. The resulting stasis of intravascular cellular flow, the hemconcentration that follows, the transendothelial migration and extravascular accumulation of inflammatory cells are all very likely to influence the degree of gingival erythema, edema and hyperplasia. ${ }^{11,12}$

The effects of ovulation induction on gingiva seemed to start very early in the infertility treatment and the severity of gingival influences was increased by the prolonged duration of the drug usage. It was found that women who were using $\mathrm{CC}$ for more than three menstrual cycles had significantly $(p<0.01)$ higher levels of gingival inflammation, bleeding than that of other test and control groups (mean of 1.96). One reason for this finding may be increased exposure time of gingiva to the elevated and sustained levels of hormones in the circulation. The traces of $\mathrm{CC}$ has already been detected in the plasma for at least 30 days $^{13}$ and can be detected in urine after 42 days ${ }^{14}$ of single application, hence it may be suggested that the drug may have a cumulative effect on both hormone levels and gingival inflammation when applied in subsequent cycles.

Elevated concentrations of estrogen and progesterone alter gingival microvascularization and result in gingival edema and increased flow of gingival crevicular fluid. Estrogen also acts as growth factor and promotes the colonization of certainly highly pathogenic bacteria, such as P. intermedia. ${ }^{15-17}$ Ovulation induction also exacerbates gingival inflammation, bleeding and GCF volume and that the usage of these drugs is strongly associated with the severity of gingival inflammation. ${ }^{18}$

It is sensible to assume that the previous periodontal disease and/ or gingival inflammation induced by ovulation drugs may affect the outcome and the success of the infertility treatment. The current considerable interest in the association of periodontal diseases and adverse pregnancy outcomes is based on the infection hypothesis, ${ }^{19}$ which claims that the microorganisms and/or their products and the inflammatory cytokines in response to microorganisms might enter the uterine cavity by the blood borne route from a nongenital focus such as periodontal tissue. The association between maternal periodontitis and preterm birth weight was suggested first by Offenbacher et al. ${ }^{20}$

In this study after scaling was done a significant decrease in the inflammatory condition of gingiva and plaque scores was observed after 1 month when compared with day 0 scores, all the values were statistically significant $(p<0.0001)$. Comparison of scores among test and control groups showed presence of inflammation in test groups after scaling which might be indication the presence of elevated levels of circulating hormones in the serum and also GCF. Although inflammation was observed in the test group the periodontal health was improved when compared with the status of gingiva at baseline.

Therefore, if low levels of plaque are established and maintained during the infertility treatment course, the effects of gingival inflammation on the success of treatment would be minimized and this requires meticulous oral hygiene and routine professional prophylaxis at the beginning of each cycle to ensure the presence of most healthy periodontium 
on ovulation day on which the ovulation induction is performed. Furthermore, if a pregnancy is achieved, the health of the periodontal tissues will also be important in terms of successful implantation of the fetus in the uterus, preterm labor and low birth weight.

\section{CONCLUSION}

The results of this study have shown that ovulation induction by drugs $\mathrm{CC}$ and Letrozole, which is the most common method in the management of infertility, exacerbates gingival inflammation. Scaling and root planing still stand as the traditional methods of controlling subgingival microflora. A single session of scaling can yield a significant reduction in the bacterial population. Gingival inflammation is risk factor for pregnancy and pregnancy outcomes, thus its control by scaling would play a main role in the successful outcome of the infertility treatment.

\section{REFERENCES}

1. Rosene MK, Keely E, Laifer SA, Lee RV. Evaluation and management of infertility in woman: the internist's role. Ann Intern Med 2000;132:973-981.

2. Vayena E, Rowe PJ, Griffin PD. Medical, Ethical and Social aspects of assisted reproduction. Report of WHO meeting. Geneva: World Health Organization 2001:3-21.

3. Roy H, Vaclav I. Ovulation induction in perspective. Hum Reprod Update 2002;8(5):449-462.

4. Zachariasen. The effect of elevated ovarian hormones on periodontal health: oral contraceptives and pregnancy. Women and Health 1992;20:21-30.

5. Parkar MH, Newman HN, Olsen I. Polymerase chain reaction analysis of oestrogen and androgen receptor expression in human gingival and periodontal tissue. Arch Oral Biol 1996;41:979-983.

6. Oht J, Erber R, Wang HL. Periodontal diseases in child and adolescent. J Clin Periodontol 2002;29:400-410.

7. Yalcin F, Eskinazi E, Soydinc M. The effect of sociocultural status on periodontal conditions in pregnancy. J Periodontol 2002;73:178-182.

8. Raber-Durlacher JE, van Steenbergen TJ, Van der Velden U, de Graaff J, Abraham-Inpijn L. Experimental gingivitis during pregnancy and postpartum: clinical, endocrinological and microbiological aspects. J Clin Periodontol 1994;21:549-558.

9. Armitage GC. Development of a classification system for periodontal diseases and conditions. Ann Periodontol 1999;4: 1-6.

10. Mealey B, Moritz A. Hormonal influences: effects of diabetes mellitus and endogenous female sex steroid hormones on the periodontium. Periodontol 2000. 2003;32:59-81.

11. Lindhe J, Branemark PI. Changes in vascular permeability after local application of sex hormones. J Periodont Res1967;2: 259-265.

12. Lindhe J, Branemark PI. Changes in vascular proliferation after local application of sex hormones. J Periodont Res 1967;2: 266-272.
13. Plouffe L Jr. Selective estrogen receptor modulators (SERMs) in clinical practice. J Soc Gynecol Investig 2000;7:38-46.

14. Moran C, Carranza LS, Noyala JE, Garcia HE, Henao G, Bermudez JA. Effect of chlomiphene citrate on ovulation after treatment withdrawl. Arch Gynecol Obstet 1998;261:117-120.

15. Raber-Durlacher JE, Van Steenberg TJ, Van der Velden U, de Graaff J, Abraham IL. Experimental gingivitis during pregnancy and postpartum: clinical, endocrinological and microbiological aspects. J Clin Periodontol 1994;21:549-558.

16. Nakagawa S, Fujii H, Machida Y, Okuda K. A longitudinal study from prepuberty to puberty gingivitis. Correlation between the occurrence of Prevotella intermedia and sex hormones. J Clin Periodontol 1994;21:658-665.

17. Kornman KS, Loesche WJ. Effects of Estradiol and progesterone on bacteriodes melanogenicus and bacteroides gingivalis. Infect Immun 1982;35:256-263.

18. Haytac MC, Cetin T, Seydaoglu G. The effects of ovulation induction during infertility treatment on gingival inflammation. J Periodontol 2004 Jun;75(6):805-810.

19. Gibbs RS. The relationship between infections and adverse pregnancy outcomes: an overview. Ann Periodontol 2001;6: 153-163.

20. Offenbacher S, Katz V, Fertik G, et al. Periodontal infection as a possible risk factor for preterm low birth weight. J Periodontol 1996;67(10):1103-1113.

\section{ABOUT THE AUTHORS}

\section{Sanjay Vasudevan}

Professor and Head, Department of Periodontics, Army College of Dental Sciences, Secunderabad, Andhra Pradesh, India

\section{J Valli Renuka (Corresponding Authors)}

Senior Lecturer, Department of Periodontics, Army College of Dental Sciences, Jai Jawahar Nagar (CRPF Road), Chennapur, Secunderabad 500087, Andhra Pradesh, India, Phone: +91-9949997247, e-mail: jvallirenuka@gmail.com

\section{Sharon Sylvia}

Senior Lecturer, Department of Periodontics, Army College of Dental Sciences, Secunderabad, Andhra Pradesh, India

\section{Radhika Challa}

Senior Lecturer, Department of Periodontics, Army College of Dental Sciences, Secunderabad, Andhra Pradesh, India

\section{Padmakanth}

Senior Lecturer, Department of Periodontics, Adithya College of Dental Sciences, Beed, Maharashtra, India

\section{Ajay Reddy}

Reader, Department of Periodontics, Army College of Dental Sciences Secunderabad, Andhra Pradesh, India 